Muséologies

Les cahiers d'études supérieures

muséologies

\title{
« Partager la connaissance, diffuser la recherche, confronter les disciplines » : auditoriums et programmation temporaire dans les musées
}

\section{Paul Salmona}

Volume 3, numéro 2, printemps 2009

URI : https://id.erudit.org/iderudit/1033571ar

DOI : https://doi.org/10.7202/1033571ar

Aller au sommaire du numéro

Éditeur(s)

Association Québécoise de Promotion des Recherches Étudiantes en Muséologie (AQPREM)

ISSN

1718-5181 (imprimé)

1929-7815 (numérique)

Découvrir la revue

Citer cet article

Salmona, P. (2009). « Partager la connaissance, diffuser la recherche, confronter les disciplines » : auditoriums et programmation temporaire dans les musées. Muséologies, 3(2), 172-181. https://doi.org/10.7202/1033571ar 
Article douze

\section{" Partager la connaissance,}

\section{diffuser la recherche, confronter les disciplines »}

\section{Auditoriums et programmation temporaire}

\section{dans les musées}

PAUL SALMONA A DIRIGÉ L'AUDITORIUM DU MUSÉE DU LOUVRE DE 1992 À 2005, APRĖS AVOIR ÉTÉ CONSULTANT AUPRÈS DE NOMBREUSES INSTITUTIONS CULTURELLES PUIS DIRECTEUR DE LA COMMUNICATION DE LA DIRECTION DES MUSÉES DE FRANCE ET DE LA RÉUNION DE MUSÉES NATIONAUX. IL INTERVIENT À L'ÉCOLE DU LOUVRE DEPUIS 2006 SUR LE THĖME \&AUDITORIUMS DE MUSÉE ET PROGRAMMATION TEMPORAIREN.ILEST ACTUELLEMENT DIRECTEUR DU DÉVELOPPEMENT CULTUREL DE LINSTITUT NATIONAL DE RECHERCHES ARCHÉOLOGIOUES PRÉVENTIVES. [paul.salmona@inrap.fr] 


\section{Le cinéma entre au musée}

La diversité des programmes proposés aujourd'hui par les auditoriums de musées trouve notamment ses prémisses dans la nécessité de montrer le cinéma que les musées commencent à collectionner dans l'entre-deux-guerres. La constitution d'une collection de films par le Museum of Modern Art de New York, à l'initiative d'Alfred H. Barr, est un jalon important dans cette évolution. Avec la création de la Film Library par Iris Barry en 1935, le cinéma entre au MoMA comme art majeur du $\mathrm{XX}^{\mathrm{e}}$ siècle. Le bâtiment de Philip L. Goodwin et Edward Durrell Stone, inauguré en 1939, comporte déjà une salle de 450 places. La présence patrimoniale du septième art dans les collections ${ }^{[1]} s^{\prime}$ accompagne d'une programmation, qui se poursuit aujourd'hui dans deux salles, sous le titre de Film Exhibitions ${ }^{[2]}$.

À Washington, la National Gallery of Art propose, depuis 1941, un programme de cinéma et de conférences, qui a pris une grande ampleur avec l'ouverture en 1978 d'un auditorium de 500 places conçu par leoh Ming Pei. C'est également à l'architecte sino-américain que l'on doit la salle de 380 places du Boston Museum of Fine Arts inaugurée en 1981. Sa programmation, dont l'origine remonte à 1958, en fait un lieu de référence pour le cinéma d'auteur sur le continent américain ${ }^{[3]}$. 
[1]

Un corpus de 22000 films à ce jour.

La création de Cinémathèque française date de 1936 et sa première salle de projection de 1948. Située

au 7 , avenue de Messine à Paris, elle n'avait que 60 places et il faudra attendre 1955 pour

voir l'institution dotée d'une salle de 260 places au 29 de la rue d'Ulm.

[3]

II propose aujourd'hui 700 séances par an

[4]

Le site Internet du Musée d'Art moderne de la Ville de Paris est muet sur cette période:

«Travaux 1971-1972 et 1992-

1994 [.... . Le plan initial fut modifié; on créa un plancher

qui coupait l'ancien hall d'entrée, une mezzanine pour les bureaux et le centre de documentation, un auditorium relié aux nouvelles salles par une passerelle à deux niveaux et un ascenseur pour desservir tous les niveaux du musée. Cet usage de l'espace, typique de l'époque, montra ensuite très vite ses contraintes. L'ARC, jusqu'alors nomade, allait s'installer dans les salles du premier étage. De la programmation,

on ne saura rien!

[5]

On peut en visionner une courte vidéo sur Youtube: $<$ Lttp://www.youtube.com/ watch? $=\mathrm{ik}|\mathrm{DW} 1-\mathrm{HLA}\rangle$.

Voir XENAKIS, lannis.

"Polytopes"; et GUY Michel. "Dix ans et la suite». In. Festival d'Automne à Paris 1972-1982. Paris: Éditions Messidor, 1982.

[7]

Dans ce domaine mal documenté, l'ouvrage de Paul ARDENNE est un précieux document : CAPC Musée 1973-1993. Paris: Éditions du Regard, 1993.

\section{Expérimentations parisiennes}

En France, dans les années 1970, le conservateur Pierre Gaudibert convie John Cage, Merce Cunningham ou Pierre Guyotat, au Musée d'Art moderne de la Ville de Paris, dans le cadre des activités de l'ARC (animation, recherche, confrontation), mais ces initiatives restent l'exception ${ }^{[4]}$. Le Polytope créé par Iannis Xenakis en 1972 dans le cadre de la première édition du festival d'Automne, au musée du Moyen Âge, dans le frigidarium des thermes de Cluny, marque l'investissement d'un espace patrimonial et muséal par une œuvre multimédia ambitieuse. Le compositeur y recourt en précurseur à l'ordinateur et à un dispositif de lasers pour produire 24 minutes d' " actions de lumière et de son " ${ }^{[51}$, associant musique électronique et sculpture lumineuse, auxquelles pourront assister 100000 personnes en 15 mois $^{[6]}$.

Enfin, concernant cette période pionnière, on doit aussi citer le travail de Jean-Louis Froment au Centre d'arts plastiques contemporains (CAPC) de Bordeaux, où une active démarche de programmation a accompagné pendant 20 ans une audacieuse politique de présentation de l'art le plus contemporain ${ }^{[7]}$.

\section{Le paradoxe du Centre Pompidou}

Ouvert en 1977, le Centre Pompidou revendique une large pluridisciplinarité. Sous l'égide de Jean-Loup Passek, de 1978 à 1997, des rétrospectives y seront dévolues à des cinémas méconnus (tchèque, slovaque, australien, brésilien, coréen...). Leur principe rappelle celui de la réunion d'œuvres par écoles nationales, qui prévaut dans nombre de musées d'art; la prééminence du cinéaste y est secondaire, en rupture avec une tradition critique française vouée aux auteurs. Aujourd'hui, la programmation tente de rendre compte de l'envahissement du champ de l'art par tous les médias de l'image animée (dispositifs cinétiques proches du pré-cinéma, film, vidéo.... ${ }^{[8]}$ et des influences réciproques des arts visuels et du cinéma, dont témoignait magnifiquement Le mouvement des images, un accrochage temporaire des collections par Philippe-Alain Michaud ${ }^{[9]}$, conservateur responsable de la collection de cinéma du Musée national d'art moderne, en 2006-2007 $7^{[10]}$. Par ailleurs, sur l'initiative de la Bibliothèque publique d'information, du Musée national d'art moderne ou de la direction du Développement culturel, le Centre Pompidou explore tout le spectre de la recherche et de la création : les « Revues parlées " abordent la littérature, la philosophie, l'esthétique, les arts plastiques, l'architecture, le design, le graphisme; les "forums de société» s'intéressent aux liens entre création et mutations sociales. Le bâtiment de Renzo Piano 


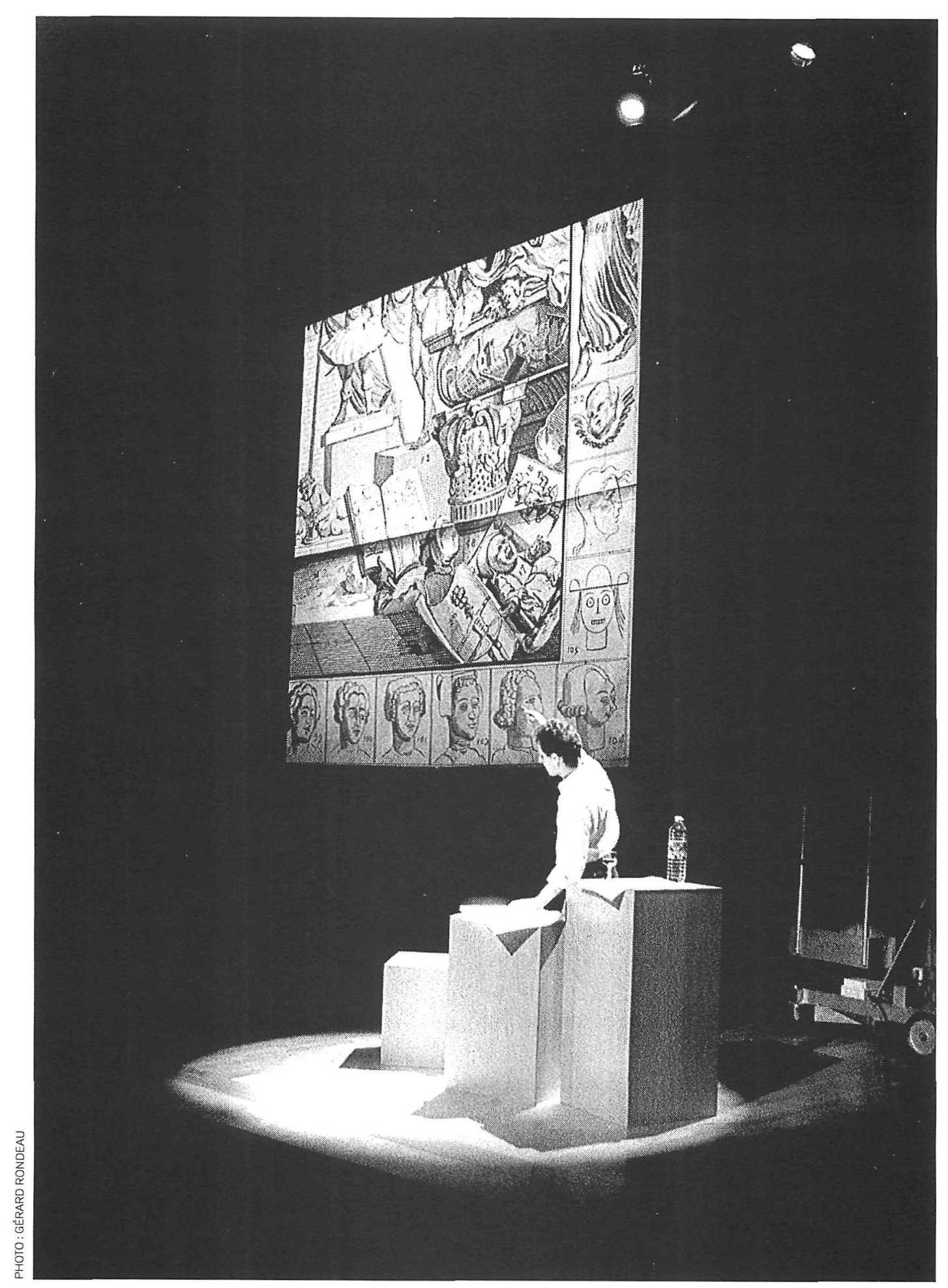

Charles Berling lit Analyse de la Beauté, de William Hogarth, le 26 octobre 1994, à l'auditorium du Louvre 
[8]

La programmation du centre propose des monographies intégrales, des programmes thématiques, du cinéma expérimental, des rétrospectives d'art vidéo, le festival "Cinéma du réel", le festival "Vidéodanse"...

[9]

"Aujourd'hui, alors que le cinéma, porté par la révolution numérique, migre des salles de cinéma vers les espaces d'exposition, il devient possible, sinon nécessaire, de reconsidérer son histoire d'un point de vue élargi (et de renouer

ainsi avec sa préhistoire),

dans ses interactions et ses prolongements avec l'ensemble des arts visuels et plastiques -

c'est-à-dire de repenser le cinéma du point de vue de l'his-

toire de l'art et dans le cadre histoire générale des représentations, et non plus simplement du point de vue restreint de

l'histoire du cinéma." (MICHAUD, Philippe-Alain. Le Mouvement des images. Paris: Éditions du Centre Pompidou, 2006.

[10]

Dans le prolongement d'une exposition comme PeintureCinéma-Peinture conçue par

Germain Viatte à la VieilleCharité à Marseille en 1989.

[11]

Voir l'entretien donné en 2004 par Serge Laurent sur le site Internet du centre: $<$ http://www.centrepompidou. fr/education/ressources/ENSspectacles-vivants/index.htm $D$.

[12]

À l'automne 2008, outre

Un dimanche, une œuvre et le cycle Tumulte dans les collections ou le Musée d'art moderne réinventé

la programmation n'aborde pas l'histoire de l'art.

[13]

Voir l'interview donné par Pierre Korzilius à Maya Prynda sur le site <ResMusica.com> et Richard Rogers, entièrement rénové après plus de vingt ans d'intensif fonctionnement, est doté de quatre salles depuis sa réouverture en 2000 ; la programmation y connaît un renouveau, notamment avec les Spectacles vivants, sous l'impulsion de Serge Laurent. Ces programmes offrent un espace à la danse, aux musiques électroniques, soulignent les liens entre les arts visuels et la scène contemporaine et témoignent d'un effacement des frontières entre "performances » issues des arts plastiques et « spectacles " venus du monde la danse ${ }^{[11]}$ ou du théâtre.

LIRCAM (Institut de recherche et coordination acoustique-musique), enfin, y propose des concerts de musique contemporaine, conformément à sa vocation. Cependant, si le centre a vocation à embrasser la « totalité» du monde contemporain, dans une programmation particulièrement riche, paradoxalement, hormis quelques conférences, l'histoire de l'art et la parole des artistes ${ }^{[12]} y$ sont aujourd'hui très discrets, notamment lorsqu'on les confronte au riche programme de la Tate Modern à Londres.

\section{Musique de chambre et cinéma des origines}

Avec près de 200 séances par saison, l'auditorium du musée d'Orsay, ouvert en 1986, porte une part fondamentale du projet originel du musée, qui ambitionnait de rendre compte de la globalité de la production artistique et intellectuelle de la période 1848-1914. Pierre Korzilius, son directeur, y aborde des genres originaux (cafés littéraires, théâtre d'ombres...) ou des petites formes qui restituent des aspects méconnus de la vie artistique à la fin du $\mathrm{XIX}^{\mathrm{e}}$ siècle et au début du XX $\mathrm{XX}^{\mathrm{e} \text { [13] }}$. La musique y est bien servie par l'acoustique de cette belle salle de 350 places due à l'architecte et designer italienne Gae Aulenti. Il y explore des répertoires de musique de chambre ou d'œuvres vocales (intégrales de compositeurs, œuvres oubliées, genres singuliers, opéras de chambre...). Des œuvres contemporaines viennent aujourd'hui enrichir les programmes des concerts à l'instar des œuvres plastiques présentées dans les salles du musée ${ }^{[14]}$. Le cinéma muet y tient aussi une place importante, en excédant très logiquement les dates charnières de la collection ${ }^{[15]}$. 


\section{Ouvrir le musée sur la ville}

$\mathrm{Au}$ Louvre, il faut attendre 1989 pour qu'une salle soit dévolue à la parole savante ${ }^{[16]}$ comme au cinéma ou au spectacle vivant. Le programme architectural de l'auditorium, défini au début des années 1980, est celui d'une salle de conférences aux ambitions modestes. Ieoh Ming Pei livre ainsi un volume magnifique équipé de $\mathbf{4 2 0}$ élégants fauteuils, mais aux capacités techniques limitées. Pourtant, Michel Laclotte, directeur du musée à partir de 1987, assigne à Guillaume Monsaingeon, chef du service de l'auditorium de 1987 à 1991, et à son équipe une ambition plus large: outre des conférences et des documentaires sur l'art, il s'agit de proposer des prolongements à la pratique muséale dans les domaines les plus variés et d'inventer une offre nouvelle qui contribue à l'« ouverture du musée sur la ville", l'un des concepts structurants du projet du Grand Louvre.

La salle devra donc offrir une tribune à l'activité de recherche des conservations, grâce à des colloques internationaux d'archéologie. Des cycles de conférences revisiteront l'histoire de l'art, en conviant de nombreux chercheurs étrangers et en convoquant des disciplines multiples pour renouveler le regard sur les œuvres. Vitrine de l'activité de production audiovisuelle du Louvre, l'auditorium présentera la part la plus intéressante de la production documentaire sur l'art et l'archéologie. Des petites formes théâtrales témoigneront des rapports de la littérature avec les arts visuels: mythologies, écrits d'artistes, récits d'atelier, journaux d'archéologues, théorie de l'art, récits de voyages, nouvelles... Des cycles de cinéma prolongeront, dans le champ du septième art, des questions fondamentales de l'histoire de la représentation. Des programmes de "musique filmée " exploreront l'interprétation musicale au XXe siècle. Tirant parti des qualités acoustiques de la salle, le Louvre commandera, de 1990 à 2008, des œuvres à des compositeurs contemporains pour des chefs-d'œuvre du muet, créés au cours de projections-concerts ${ }^{[17]}$. Un programme de musique de chambre permettra de découvrir de nombreux jeunes interprètes de toutes nationalités.

Avec près de 300 manifestations par saison, l'auditorium proposera un programme excédant souvent les époques représentées dans les collections et élargissant le spectre des disciplines du musée ${ }^{[18]}$. La richesse de cette offre contribuera à transformer l'image du Louvre et à réconcilier le musée avec un public de proximité, dont la fréquentation s'est fortement accrue depuis 1989 [19], séduit par ces "goûts réunis " [20].

\section{[14]}

Notamment la série

Correspondances introduite par Serge Lemoine en 2004. [15]

La bascule du muet au parlant s'opère dans les années 1928-1930.

\section{[16]}

Si l'on excepte, par construction, les programmes de l'École du Louvre.

[17]

Cycle intitulé Cinéma muet en concert.

[18]

Voir SALMONA, Paul.

"L'aventure de l'Auditorium *

In. "Le Louvre dans tous ses

éclats", Revue des Deux

Mondes, septembre 1999.

[19]

Les Français représentaient

$10 \%$ des 3 millions de visiteurs du Louvre en 1989, soit 300000 visiteurs, et $31 \%$ des 8,3 millions de 2007, soit 2,5 millions de visiteurs, en augmentation de $800 \%$. II serait évidemment intéressant de connaître la part spécifique des Franciliens, public le plus directement concerné par les programmes de l'auditorium, dont la fréquentation se situe autour de 70000 spectateursauditeurs par saison. [20] Pièces de François Couperin composées en 1724, dont le titre reviendra de façon emblématique pour désigner l'«esprit” de la programmation de la salle. 


\section{Rendre compte du patrimoine immatériel}

C'est aussi le projet muséal qui est en jeu dans les auditoriums de musées dont la vocation dépasse la seule présentation d'une collection d'œuvres d'art. Au Musée d'art et d'histoire du judaïsme, ouvert en 1998 dans l'hôtel de Saint-Aignan à Paris, les architectes Catherine Bizouard et François Pin ont évidé la cour pour y loger un auditorium de 182 places. Son programme très actif, conçu par Corinne Bacharach, aborde les dimensions les plus diverses de la culture et de l'identité juive par le biais de la musique, de la littérature, du théâtre ou du cinéma. C'est un dispositif essentiel dans ce musée dont l'ambition est de rendre compte des cultures et de l'engagement des juifs dans la cité, du Moyen Âge à nos jours.

De même, le musée du quai Branly ne se limite pas à la présentation de ses collections ethnographiques. Le bâtiment de Jean Nouvel, ouvert en 2006, est pourvu de trois salles ${ }^{[21]}$. Leur très active programmation contribue à la diffusion de la recherche et rend compte de la diversité des formes d'expression artistiques, notamment musicales, des peuples dont les cultures sont représentées dans le musée. Sous l'égide de Catherine Clément, l'université populaire du quai Branly y aborde sans tabous l'histoire coloniale.

\section{Dans les régions}

Avec la transformation du réseau des musées français, particulièrement marquée dans la décennie 1990-2000, le modèle a essaimé en régions. Si les musées n'y disposent pas de moyens aussi importants que ceux des musées parisiens, ils se sont néanmoins dotés de salles qui jouent un rôle parfois sans équivalent dans la vie culturelle des métropoles régionales.

L'auditorium des musées de Strasbourg est ainsi un lieu de référence pour les musiques d'aujourd'hui (expérimentales, électroniques, improvisations...). Ouvert en 1998 dans le Musée d'art moderne et contemporain conçu par l'architecte Adrien Fainsilber, cette salle de 138 places offre parallèlement son écran au cinéma expérimental et au documentaire. Son programme original, conçu par Patrick Javault ${ }^{[22]}$, comporte conférences, colloques et performances dans près de 100 séances par saison.

Les musées de Grenoble, de Lyon, de Marseille, de Nice ou de Nîmes proposent également des conférences, des programmes de cinéma ou des concerts de musique de chambre. 


\section{Des moyens nouveaux}

Ces auditoriums bénéficient parfois de budgets importants ${ }^{[23]}$ et sont programmés par des équipes qui réunissent un très large spectre de compétences. Cette relative autonomie leur permet d'appliquer, dans les grandes «machines» muséales, des logiques plus réactives que celles de la gestion d'une collection permanente, adaptées aux exigences du spectacle vivant et à la relation avec des publics sans cesse à reconquérir. On y trouve souvent, à une échelle réduite, la structure d'organisation des théâtres ou des salles de concerts (programmation, production, relations avec le public et régie technique). En effet, le « temps» de ces lieux est différent de celui des musées dont la gestion s'inscrit dans la longue durée.

Ces auditoriums ont introduit des problématiques nouvelles - qui prolongent et amplifient la prise en compte des publics, comme le souci de la médiation - encore embryonnaires dans les années 1980. Mais ils leur conferent une ampleur particulière et une spécificité dont témoigne la création de services autonomes des services culturels ${ }^{[24]}$. Ces activités nouvelles resurgissent dans les collections et les programmes temporaires colonisent progressivement les salles du musée, temporairement ou régulièrement dévolues à des activités spectaculaires : concerts dans la salle des fêtes et dans la nef du musée d'Orsay, lectures et danse dans les salles et les cours du Louvre, etc.

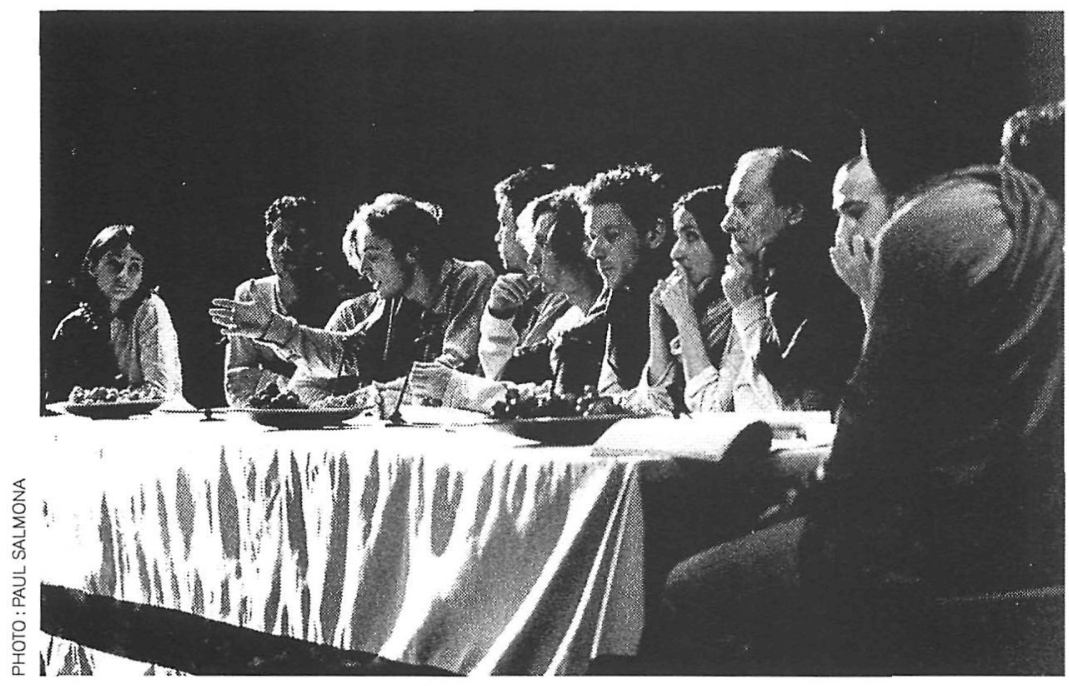

Treize comédiens, sous la direction de Brigitte Jaques, lisent l'intégrale de l'Énéide de Virgile le 11 avril 1999, de 10 heures à 2 heures du matin, à l'occasion du $10^{\mathrm{e}}$ anniversaire de l'ouverture du hall Napoléon et de l'auditorium du Louvre.

\section{[23]}

Près de un million d'euros au Louvre en 2007 pour les seules dépenses de production de l'auditorium.

[24]

Au Louvre, la vocation de la salle et l'organisation adoptée susciteront une incompréhension durable dans les conservations, mais seront confortées par les présidents successifs. 


\section{Une pluralité des discours}

Cette ouverture est désormais revendiquée par nombre de musées. Il suffit de consulter les documents programmes de chaque institution pour constater la place prépondérante prise par la programmation, sa richesse et sa diversité. On assiste ici à une transformation profonde de la vie des musées.

[25]

Sur la genèse des auditoriums des musées d'Orsay et du Louvre, on pourra néanmoins utilement se référer à LACLOTTE, Michel. Histoires de musées. Paris: Scala, 2003, p. 226-227 et 263-266. Ce ne sont plus seulement des lieux de conservation, d'étude, de restauration et de présentation des collections, conçus pour la délectation des connaisseurs. Ils deviennent les lieux du partage de la connaissance, de la diffusion de la recherche et de la confrontation des disciplines, de la mise en relation des arts et de la diversité des discours, de la résonance de la création contemporaine avec les œuvres du passé.

Contrairement aux augures élitistes qui voyaient, dans le mouvement récent de transformation des musées, une concession aux logiques du marketing et une prostitution des collections au tourisme de masse, les musées démontrent un souci de renouvellement, d'ouverture et d'exigence. Au dispositif, fondamental mais rigide par essence, de l'accrochage permanent des œuvres répondent désormais des dispositifs discursifs pluriels. Mais, éphémères par nature et sans tradition d'archivage, ils restent aujourd'hui mal documentés et peu étudiés ${ }^{[25]}$.

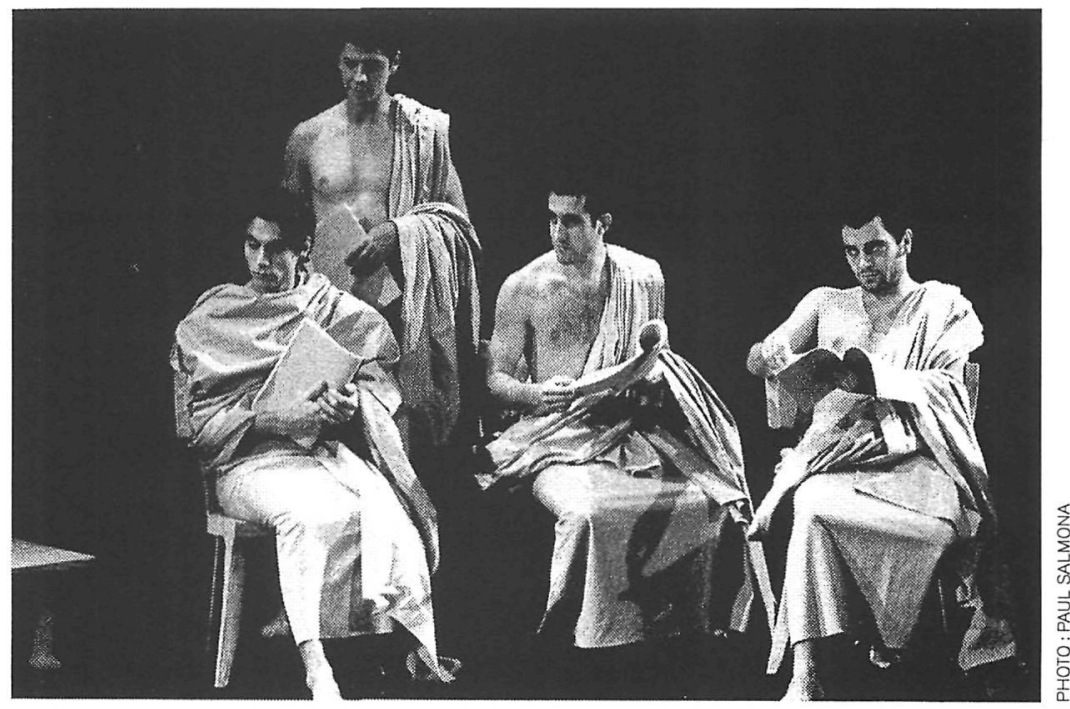

20 comédiens, sous la direction de Brigitte Jaques, lisent l'intégrale de l'Odyssée d'Homère les 21 et 22 octobre 2002, à l'auditorium du Louvre. 


\section{Summary}

[Translated by Melissa Rose]

Over the last 20 years, the diversification of auditoriums' programming has become one of the major aspects of museums' expansion. Since the early 1930s, the Museum of Modern Art (MoMA), whose collection spans mainly from the essence of filmmaking, comes with a public programme entitled "Film Exhibitions." Since 1941, The National Gallery of Art in Washington (DC) houses a gallery that has greatly expanded with the opening of its huge auditorium in 1978. In France, during the 1970s, similar initiatives have taken place at the Musée national d'art moderne in Paris or the Centre d'arts plastiques contemporains in Bordeaux. First opened in 1977, not only is the Centre Pompidou devoted to all forms of creations such as literature, philosophy, plastic arts, design, dance, music, but it also dispels the myth between performances and shows. Opened in 1986, the auditorium of the Musée d'Orsay carries a fundamental aspect of the museum's project, which encompasses the whole of artistic and intellectual production covering the period 1848-1914. At the Louvre, Michel Laclotte completed the museum's room which was built under the pyramid in 1989 with the idea of creating an "open window on the city," based on archaeology, history of art, documentaries, reading, silent movie concerts, chamber music. Opened in 1998 in Paris, the Musée d'art et d'histoire du judaïsme retraces the development of Jewish history, cultural heritage, and traditions through music, literature, theatre, or cinema. The three rooms of the Musée du quai Branly, which opened in 2006, account for diversity in artistic expression of non-occidental civilizations. During the 1990-2000 decade, that model has spread out in areas where museums play an important role in the lives of major cities such as Strasbourg, Grenoble, Lyon, Marseille, Nice, or Nîmes. These auditoriums introduced new embryonic issues in the 1980s, which reappeared throughout the collections, and gradually "colonized" museums' rooms. Now museums are not only sites of "connoisseurship," but that of knowledge sharing, research dissemination, discipline confrontation, that of existing relationships between arts and the variety of speeches, and of the echo of contemporary creations with artworks from the past. 